\title{
The Electronic Health Record Paradox: Observations of a GI Consultant
}

\author{
David A. Lieberman ${ }^{1}$
}

Published online: 6 October 2015

(c) Springer Science+Business Media New York 2015

"It was the best of times, it was the worst of times," wrote Charles Dickens in the Tale of Two Cities. He could well have been talking about the electronic health record (EHR) and medicine in the twenty-first century. My career has spanned a remarkable transformation from a paper medical record system to an electronic system. To understand this transformation, we must recall the common frustrations of the paper era. Handwritten, illegible records, lost laboratory test results and reports, prescription errors, and little systematic documentation of anything were the norm. Reflecting back on twentieth century medicine, it seems remarkable that we were able to muddle through and help our patients. This may have been a tribute to the resiliency of our patients, but there may be another explanation. I think we spent more time with patients, focused more on the medical history, had more continuity of care with fewer "hand-offs," and did not rely on a "cut-and-paste" method of constructing the patient narrative. This is my thesis, and I fear it makes me seem old and out of date, so let me elaborate.

I am hardly a technology Luddite. In the hope of introducing a totally electronic medical record, I helped develop an electronic report generator for endoscopy reporting to enable endoscopic research (Clinical Outcome Research Initiative, CORI). I envisioned that the easy availability of laboratory and testing data, prior records, and notes would reduce the time physicians spend trying to locate key records and enable them to spend more time in

David A. Lieberman

lieberma@ohsu.edu

1 Division of Gastroenterology and Hepatology, Oregon Health and Science University, L461 3181 SW Sam Jackson Park Rd., Portland, OR 97239, USA face-to-face contact with patients. "Smart" systems would reduce medical errors in prescribing and testing. A medical problem might be identified, and evidence-based algorithms would appear to help direct the clinician toward the most effective approach to the issue. Clinical reminders would be embedded so that crucial anniversary dates for preventive measures would easily be recalled. It is exciting that so many of these features are now in place.

Nevertheless, the electronic era has not lived up to its potential, due in part to failures of the electronic system and in part to human adaptation to the system.

\section{The Electronic Factors}

Since the first priority of hospital systems at the dawn of the EHR era was to capture billable events, facilitating billing and coding was the focus of the nascent electronic record developers, a priority that as a scientist I found frustrating and disappointing. I wanted to be able to link patient demographics, history, and laboratory and test results to better understand disease states and help my patients. I wanted to be able to add on novel instruments that might help identify high-risk patients who would need more intensive management of their medical conditions. Generally, add-ons are simply not allowed in today's EHRs, or placed into a queue, where they disappear. I want to be able to use electronic tools to measure quality metrics. All of these analytics may yet appear, but this has not been the priority of either the developers of software or the hospital systems that buy them (at great cost). Once purchased, an EHR is too great an investment to discard, even when it fails to live up to the hype about flexibility in the initial sales pitch. 
I dreamed that the systems would not be in "silos," that a common language (like HL7) would enable systems to talk to each other across medical centers and continents. Since patients do not receive their care in one place, we are still challenged to obtain reports, laboratory data, and imaging results from other healthcare systems, which when unavailable often results in unnecessarily repeating tests. If electronic systems were built to communicate seamlessly among each other, such problems would not exist. Despite attempts to build a common language in medicine, we are still struggling with a virtual Tower of Babel that impairs communication across systems, which is a big disappointment.

How can this get better? We should demand quality assurance from the EHR, just as we now do for other aspects of medical practice. What would an EHR quality program look like? The system would enable communication with other EHRs and do away with the Tower of Babel in medical informatics. It would permit the addition of well-validated add-on features. A great example is a tool for easily obtaining a family history of colorectal cancer to determine if the patient might fall into a high-risk group. As wonderful and simple as this tool may be, it is not currently feasible to embed these data into the medical record. The only obstacle is proprietary restrictions. This should change, and there are many other examples that would enhance the value of the EHR. Systems designed primarily for coding and billing are not necessarily the best systems to enable high-quality patient care and patient analytics. To fully utilize the power of these tools, we need to encourage and cajole the software engineers to put as much time into patient care analytics as they do into billing and coding.

\section{The Human Factors}

The impact of electronic systems on the training of doctors is substantial. On the positive side: information about the patient is readily available, provided it was generated within the health care system. This saves considerable time that I wasted during my residency trying to find laboratory results, or understand the trends in laboratory changes. Images show up on the computer screen, electronic notes and orders are legible, and prescription errors are more readily detected.

Nonetheless, there is also a dark side of the EHR that I have observed as a gastrointestinal (GI) consultant in an academic medical center. The inpatient medical record has become a repository of waste. I defy anyone to read through progress notes in an inpatient record over $48 \mathrm{~h}$ and figure out if there is or is not progress. The documentation requirements compel clinicians to include key elements (for coding), however redundant they might be. The cutand-paste capabilities of the electronic system result in what I term "chart pollution": notes become littered with irrelevant laboratory data, medication data, and vital signs, all of which are easily accessible elsewhere. The notes are long and unreadable. Why do we ask our trainees to put this information in their notes, when all we really want is an analysis of key relevant laboratory and test results in the context of the patient's course? As a consultant, trying to understand 24-h events in the life of an inpatient, I find these notes to be worse than useless, because they obscure the really important data.

Worse than worthless notes is the amount of time that housestaff spend producing these notes, time which is spent in front of computer screen rather than with the patient. I have not seen good data on this, but I suspect that the actual face time of interns and residents with inpatients has declined in the electronic era. At least when I was starting intravenous lines and transporting patients to radiology as an intern, I was in constant contact with the patient. And that contact is valuable. There is not only the verbal contact, but also nonverbal cues derived from getting to know our patients and understanding the impact of an illness on their lives. I fear that we miss some of these cues when we spend more hours in front of the computer screen than with the patient.

In a world with prudent resident hour limits and frequent "hand-offs" of care, who really gets to know the patient and his or her illness? The hand-off becomes crucial, and in a cut-and-paste world, information (correct or incorrect) is transmitted to the next provider. I see little effort to identify and correct mistakes in the EHR when they occur, and even less time spent going back to the patient for clarification.

In the outpatient setting, the pressures for documentation and haste are even more intense. Primary care providers and specialists have to spend what little time is scheduled for patients performing onerous documentation for proper coding. In a typical office or clinic, physicians are often positioned in front of a computer screen and have little eye contact with the patient. Non-verbal cues may be lost if the provider is staring at the screen or typing on the keyboard during the visit. There is tremendous pressure to complete the visit, record the note, and move to the next waiting patient. Opportunities for patients to express their goals and concerns may become secondary to these pressures. Cutting and pasting and use of defaults becomes the norm to save time but may lead to perpetuation of errors. As a consultant, I see many of these default notes from primary care providers that document a negative GI review of systems, despite referral for GI complaints.

How can we improve human adaptation to the electronic systems? We can start by producing meaningful notes. It is ironic that we are mandated to document "meaningful use" 
of the EHR, but there is no mandate to do it wisely. The medical note should include relevant elements of a medical history and physical examination and summaries of relevant laboratory test results, and focus primarily on an assessment and plan. Redundant cutting and pasting should be banned because this obscures what is really important and renders the note unreadable. Pasting a list of all medications and laboratory results into the note does not make a note more meaningful. Progress notes should communicate something about progress or lack thereof. Clinic notes should provide meaningful updates of problems. This is all common sense and can be changed. We need to continue to improve our hand-off skills so that the patient and his or her problem(s) is not lost in the process.

In the outpatient setting, the computer can create an emotional distance between the physician and the patient. We need to recapture that intangible benefit of eye contact with our patients; this means taking our eyes away from the computer screen. When I see patients in clinic, I always do three things: I ask the patient about his or her goals for the visit, so that I understand what they are hoping for; I turn and face the patient and make nearly constant eye contact when I am taking the medical history; and I look for nonverbal cues. I always ask patients if they have any other concerns and questions before they leave and do so while looking straight into their eyes. In the clinic, we can create educational opportunities that take advantage of the strengths of the EHR. I love having images on screen that I can show the patient. I regularly review computed tomography scans during a clinic visit and find that, with a little explanation, patients can understand what I am showing them; this takes away a little of the mystery of medicine. There are new tools for patients to enter their medical history on a pad device before coming to clinic. The questions follow a logical flow, and are provided at a sixth-grade reading level; a narrative is produced for the provider when the patient appears. The provider can focus on clarifications. I like this because clinic visits can be intimidating and foreboding for some patients who have "white-coat" anxiety and fail to provide important information. The medical pad can be empowering for patients. We can make the computer a better tool that enriches the clinic visit, rather than one which takes our eyes and minds away from the patient.

The EHR is not the enemy, although it could be a better friend. There are amazing possibilities. We need to insist on quality assurance metrics for our electronic tools. Ultimately, the electronic tools depend on the humans using them-and we can also get better.

To paraphrase Cassius in Shakespeare's Julius Caesar:

The fault, dear Brutus, is not in our stars [or our computers]

But in ourselves...

Compliance with ethical standards

Conflict of interest None. 